\title{
Index for Volume 13 of Molecular Plant-Microbe Interactions
}

\section{AUTHOR AND SUBJECT INDEX}

Acland, D. P., 54

Aeschbacher, R. A., 763

Aftergoot, E., 673

Agrobacterium spp.

-A. rhizogenes, ORF8 gene product, tryptophan 2-monooxygenase activity, 787

- A. tumefaciens, on maize, apoptosis induction, 649; on soybean, T-regions and Amadori opines, vir helper plasmid, 1081

Aguilar, I., 421

Aguilar, O. M., 1228

Ahn, J.-H., 80

Aivalakis, G., 14

Alfalfa (see also Medicago spp.)

—nodule development, organogenetic pathways, molecular markers, 96

-nodules, intracellular, 1204

-Phoma medicaginis, resistance and resveratrol-glucoside, 551

Ali, G. S., 847

Alias, E., 316

Alkaloid, Lolium, grass-endophyte mutualism and aphids, 1027

Alnus glutinosa, Casuarina glauca homolog, protease gene, 113

Alternaria spp.

-A. alternata: cyclic peptide synthetase gene, cloning, toxin synthesis and pathogenicity, 742; on Japanese pear, AK-toxin biosynthesis and pathogenicity, 975; matingtype genes, 1330

-A. solani, on potato, inhibition by synthetic peptides, 847

Andersson, R. A., 384

Andrews, D. L., 781

Antoun, H., 1271

Aphanomyces euteiches, on pea roots, symbiosis and bioprotection, 238

Apple, Alternaria alternata, toxin synthesis and pathogenicity, 742

Arabidopsis

-cell death and pathogen resistance, salicylic acid dependence, 877

-Turnip crinkle virus on, coat protein and AVR factor, 1015

Arabidopsis thaliana

-Cauliflower mosaic virus, movement protein gene mutation, host suppressors, 512

- defense gene expression, enzymes from Erwinia carotovora, 430

-hypersensitivity in mutants, gene-for-gene resistance, 277

-Pseudomonas syringae, defense response and salicylic acid, jasmonic acid signal, 503

-root explant cultivation, T-DNA transfer and integration, 658

— sugar beet cyst nematode, mRNA abundance, changes, 309

-Tobacco mosaic virus on, cell-to-cell movement and susceptibility, 1139

Arie, T., 1330

Astua-Monge, G., 911, 1365

Auguy, F., 113
Auriac, M.-C., 1204

Auxin, insensitivity mimic, pathogen-induced gene, 23

Avrova, A. O., 1092

Bae, C. G., 470

Bahro, R., 703

Bai, J., 1322

Baidyaroy, D., 88

Bailey, A. M., 538

Bailey, M. J., 1293

Bakker, J., 830, 1121

Barash, I., 683

Barker, H., 125

Barley, powdery mildew, germ tubes, cAMP pathway control, 494

Barras, F., 882

Batut, J., 483

Bauer, W. D., 637

Baulcombe, D. C., 599

Baum, T. J., 309

Bean common mosaic virus, on bean, hypersensitivity and vascular necrosis, $I$ gene, 1266

Bean dwarf mosaic virus

-on Phaseolus vulgaris, BV1 protein and hypersensitivity, 1184

-on tomato, Tomato mottle virus relation, geminivirus movement proteins, 297

Bécard, G., 693

Beck, D. L., 962

Beer, S. V., 1251

Beet necrotic yellow vein virus, movement proteins P42, P13, P15, plasmodesmata association, 520

Bennett, A. B., 942

Benning, C., 666

Bent, A. F., 277, 1081

Beringer, J. E., 228

Bernal, A. J., 568

Bertrand, H., 88

Beyer, K., 763

Bhattacharyya, M. K., 563

Bindschedler, L. V., 538

Bindslev, L., 494

Biological control, Pseudomonas chlororaphis for tomato foot and root rot, root colonization, 1340

Birch, P. R. J., 1092

Bisseling, T., 107

Bittinger, M. A., 1019

Blankenship, J. D., 1027

Bloemberg, G. V., 1163, 1170, 1340

Blumeria graminis, on barley, germ tubes, cAMP pathway control, 494

Boch, J., 1312

Bogusz, D., 107, 113

Boistard, P., 483, 1204

Boller, T., 763

Bonanomi, A., 763

Bonas, U., 911

Bonfante, P., 1109

Bonilla, I., 129

\section{ERRATA PAGE NUMBERS IN ITALICS}

Botrytis cinerea

-on fruit, postharvest decay, hexapeptide activity, 837

-pathogenicity and BMP1 gene, 724

Boucher, C., 259

Bouzoubaa, S., 520

Bové, J. M., 1145

Bowyer, P., 538

Bras, C. P., 475, 1163

Brassica napus, Turnip mosaic virus, cylindrical inclusion gene and pathogenicity, 1102

Brewin, N. J., 413, 715, 778, 922

Brito, B., 259

Broekaert, W. F., 54

Brom, S., 1283

Brome mosaic virus, on tobacco, virus recovery, complementation, and RNA-2specific silencing, 247

Brommonschenkel, S. H., 1130

Bromovirus, on cowpea, protein conditions, movement, and pathogenicity, 1195

Budde, I. P., 951

Buee, M., 693

Bush, L. P., 1027

Bushnell, W. R., 159

Byzova, M., 309

Cabanes, D., 483

Callaway, A. S., 512

Campbell, M., 724

Candresse, T., 316

Capsicum spp., Tomato spotted wilt virus, resistance gene, genetic mapping of locus, tomato comparison, 673

-C. аппиит: bacterial hypersensitivity, resistance and avirulence gene, 911; Xanthomonas campestris, pathogenesisrelated cDNA genes, 136

Carlile, A. J., 538

Carr, F., 962

Carrot, hairy roots, exudate effect on Gigaspora spore germination, 693

Casuarina glauca

-prenodule cell display, 107

- protease gene, nodule specific, homolog, 113

Cauliflower mosaic virus

-on Arabidopsis thaliana, movement protein gene mutation, host suppression, 512

- gene VI agroinfiltration, hypersensitivity in tobacco, 1275

Cercospora beticola, beticolins, nonpeptidic, polycyclic molecules from, ion channelforming toxins, 203

Cermola, M., 733

Cha, C., 1081

Chang, J. H., 568

Charon, C., 62, 617

Chen, J., 869

Chen, Z., 183, 1312

Cheong, Y. H., 470

Chin-A-Woeng, T. F. C., 1177, 1340

Publication no. M-2000-1025-010 
Cho, M. J., 470

Choi, S.-H., 1322

Choi, Y. J., 470

Ciuffetti, L. M., 456

Cladosporium fulvum, on tomato, induced necrosis, avirulence gene analysis, 439

Clardy, J., 1019

Clark, D., 1380

Clarkson, J. M., 538

Clavibacter michiganensis, on tomato, plasmids encoding genes for virulence, 703

Clough, S. J., 277, 1081

Cochliobolus carbonum, on maize, TOX2 chromosome, meiotic instability, 80

Cole, A. B., 1275

Collavino, M. M., 1228

Colletotrichum spp.

- C. gloeosporioides: pectate lyase, C. magna pathogenicity promoted by, 887 ; on Stylosanthes guianensis, pathogenicity gene and hypersensitivity, 929

$-C$. graminicola, mutants, restriction enzymemediated integration, 1355

-C. lagenarium, MAP kinase gene, pathogenesis regulation, 374

-C. lindemuthianum, on bean, resistance inheritance, trait loci, 287

Collmer, C. W., 1266

Comparini, C., 43

Conejero, V., 23

Cook, D. R., 763

Cooke, D. E. L., 1092

Cooper, R. M., 538

Corn (see Maize)

Cover photo

- Globodera rostochiensis, in situ hybridization, August

-Nicotiana benthamiana, Agrobacteriummediated transient expression, April

-Pseudomonas syringae on tomato, necrosis and functional genes, May

- Rhizobium in white clover root, fluorescent protein, February

-Rhizobium leguminosarum on red clover root, cortical cell arrangement, March

- Rhizobium leguminosarum on vetch, nod genes, July

- tobacco, hypersensitive phenotypes, September

- tobacco mosaic virus, on tobacco leaves, transgenic plants, January

-Turnip mosaic virus, cylindrical inclusion bodies, in Brassica, October

-white clover, nodule initiation, specific markers, June

Cowpea, bromovirus, protein and pathogenicity, host-specific movement, 1195

Crespi, M. D., 96, 617

Cryphonectria parasitica, population, transmissible mitochondrial hypovirulence, 88

Cucumber, anthracnose, MAP kinase gene and pathogenesis regulation, 374

Cucurbit, Colletotrichum spp., pathogenicity promoted by pectate lyase, 887

Culver, J. N., 1139

Dahiya, P., 778

Dahlia merckii, defensin binding site, fungus protection, 54

Dalvin, S., 494

Danet, J.-L., 1145

Dardick, D. D., 1139

Dávalos, A., 1283
Davis, M. J., 911, 1365

Day, R. B., 1053

Dean, R. A., 1214

de Billy, F., 1204

de Bruijn, F. J., 1228

de Bruxelles, G., 325

De Buck, S., 658

Dekkers, L. C., 1177, 1340

Delbos, R.-P., 316

DelGrosso, L., 1015

Dellagi, A., 1092

Del Sorbo, G., 43

de Luca, N. G., 228

Dempsey, D. A., 1015

Denny, R. L., 1053

De Oliveira, J. C. F., 287

Depicker, A., 658

De Wilde, C., 658

De Wit, P. J. G. M., 439

Díaz, C. L., 268

Dimou, M., 987

Ding, B., 599

Dinoor, A., 887

Dioh, W., 217

Di Pietro, A., 359

Ditengou, F. A., 151

Djordjevic, M. A., 170, 995

Downie, J. A., 754

Dreier, J., 703

Dron, M., 287

Dry, I., 529

Dudler, R., 342

Dufresne, M., 1301

Duhoux, E., 107, 113

Dumas-Gaudot, E., 238

Durner, J., 1380

Egan, J. D., 781

Eichenlaub, R., 703

Ellis, R. J., 1293

Ellis, T. H. N., 715

Elm, Ophiostoma quercus on, nonpathogen, hydrophobin cerato-ulmin effect, 43

Emerson, S. J., 962

Emons, A. M. C., 1384

Engelbertink, J., 1121

England, R., 1228

Epichloë, grass endophyte, alkaloid protection against aphids, 1027

Erhardt, M., 520

Eriksson, A. R. B., 384, 447

Erratum, vol. 13, no. 5, 2000, 796

Erwinia spp.

-E. amylovora: harpin from, tobacco mitochondria affected by, 183; hrp gene regulation and protein secretion, 1251

-E. carotovora: cell wall-degrading enzymes, defense gene expression in Arabidopsis, 430; endopolygalacturonase production, virulence, two-component regulatory system, 447 ; on potato, gene encoding transcription factor, endochitinase coregulation, 1092; on potato, inhibition by synthetic peptides, 847; quorum sensing, expREcc role, 384

- E. chrysanthemi: infection and role of $\mathrm{H}_{2} \mathrm{O}_{2}$, 421; $\mathrm{pH}$ effect, environmental signal, pel gene duplication, 882

- E. herbicola, on gypsophila, gene restriction, pPATH plasmid, 683

Escherichia coli, pVS1 replicon, cloning vector, 232

Eucalyptus spp., Pisolithus tinctorius on, hypaphorine and auxin relation, 151
Ezra, D., 683

Faccio, A., 1109

Fang, E. G. C., 1214

Farrand, S. K., 1081

Favre, R., 733

Fedorova, E., 733

Feldrügge, M., 1034

Fengler, K. A., 277

Flego, D., 447

Flemetakis, E., 14, 987

Forster, R. L. S., 962

Foster, G. D., 1102

Fouilloux, G., 287

Franche, C., 107, 113

Frary, Amy, 1130

Frary, Anne, 1130

Freeman, S., 887

Frey, P., 259

Freydl, E., 342

Frugier, F., 96

Frutz, T., 107

Fujita, M., 1195

Fujita, Y., 1195

Fulbright, D. W., 88

Furusawa, I., 374, 1195

Fusarium spp.

$-F$. graminearum, on wheat, defense response genes, induction in spike, 159

$-F$. oxysporum: exopolygalacturonase from, pgx4 encoding, 359; mating-type genes, 1330

García-Maceira, F. I., 359

García-Olmedo, F., 421

García-Rodríguez, F. M., 583

Garnier, M., 1145

Garrido-Ramirez, E. R., 1184

Gaurivaud, P., 1145

Gebhardt, C., 402

Geffroy, V., 287

Geiger, O., 666

Gene

-avirulence, Xanthomonas oryzae, pathogen aggressiveness, 1322

- avr, Pseudomonas syringae, on tomato, 1312

-Avr9/Cf-9 and Avr4/Cf-4, Cladosporium

fulvum on tomato, 439

-avrPto, Pseudomonas syringae in tomato: growth and necrosis, 568; mutations, 592

- BMP1, Botrytis cinerea pathogenicity gene, 724

- CgDN3, Colletotrichum gloeosporioides on Stylosanthes guianensis, hypersensitivity, 929

-chitinase, in Medicago truncatula, expression in mycorrhizae, nodulation, and infection, 763

- Crg, bean rust, analog cluster, 1237

-ENOD40, in Lotus japonicus, embryonic tissue expression, 987

- hrp, in Erwinia amylovora, regulation, 1251

-LjSym4, Lotus japonicus and symbiosis of root epidemics, 1109

—magB, mutagenesis on rice, 1214

-mating-type, Fusarium oxysporum and Alternaria alternata, 1330

-multiresistance, in Rhizobium etli, 572, 796

—nfe, Sinorhizobium meliloti, gene comparisons, 583

- purMN, Rhizobium leguminosarum, siderophore production, 228

- pyruvate dehydrogenase, from Sinorhizobium meliloti, induction, 483 
-regulatory, $h r p B$ and $h r p G$, in Ralstonia solanacearum, 259

- sss colonization, tomato-Fusarium complex, biocontrol aid, 1177

$-S w-5$ and $M i$ resistance, 1130

- tomatinase, Septoria lycopersici on tomato, 1301

-TuRB01, on Brassica, Turnip mosaic virus relation, 1102

-ubc4 and $u b c 5$, in Ustilago maydis, growth effect, 781

-Xv4-vrxv4, interaction between Xanthomonas and wild tomato, 1365

Genin, S., 259

Genre, A., 1109

Gepts, P., 287

German-Retana, S., 316

Gianinazzi, S., 238

Gigaspora spp., spore germination from carrot root exudates, 693

Gilbertson, R. L., 297, 1184

Gilmer, D., 520

Girard, L., 1283

Glazebrook, J., 503

Globodera rostochiensis

-on potato: auxin, induction and morphogenesis, 1121; pathogenicity factors, identification strategy, 830

—in situ hybridization, cover photo, August

Goh, M., 869

Gold, S. E., 781

Golem, S., 1139

González-Candelas, L., 837

González-Pasayo, R., 572, 796

Görnhardt, B., 32

Goudet, C., 203

Goverse, A., 1121

Granado, J., 342

Grasso, D. H., 1228

Greenberg, J. T., 877

Greve, L. C., 942

Grimsley, N. H., 6

Groenink, W., 830

Gross, J. A., 1019

Grube, R. C., 673

Guerreiro, N., 995

Guilley, H., 520

Gupta, V., 503

Gurr, S., 494

Györgyey, J., 62

Gypsophila paniculata, gall formation, gene restriction from pPATH plasmid, 683

Haas, D., 232

Hahn, M., 629

Hall, A., 494

Hall, T. C., 247

Hämäläinen, J. H., 402

Hamer, J. E., 374, 892

Hammond, R. W., 903

Hammond-Kosack, K., 465

Han, C., 470

Handelsman, J., 1019

Hansen, G., 649

Hart, J. K., 309

Harteveld, M., 475

Hasebe, A., 892

Hassa, P., 342

Hatfield, J., 929

Haverkamp, J., 808

Hayashi, N., 892

He, P., 592

Heeb, S., 232

Heikinheimo, R., 384, 447
Heilbronn, J., 1092

Helder, J., 830, 1121

Hermsmeier, D., 309

Heterodera spp.

- H. avenae, on wheat, resistance gene analogs, 334

-H. schachtii: on Arabidopsis thaliana, mRNA abundance, 309; auxin mediation, induction and morphogenesis, 1121

Hipskind, J. D., 551

Hiraga, S., 210

Hirano, S. S., 1263

Hoffmann, K., 673

Honma, M., 210

Hou, Y.-M., 297

Howell, S. H., 512

Huang, Z., 512

Huber, D. H., 88

Hugouvieux, V., 1301

Hwang, B. K., 136

Hypersensitivity

- Arabidopsis to Pseudomonas syringae, 277

-Bean dwarf mosaic virus on bean, 1184

- Pseudomonas syringae on Nicotiana tabacum genotypes, 1346

- tomato $C f-4$ and $C f-9$ genes in Nicotiana spp., 465

Hyytiäinen, H., 384

Ito, H., 210

Itoh, Y., 232, 742

Iyer, L. M., 247

Jahier, J., 334

Jahn, M., 673, 1266

Jahr, H., 703

Jauneau, A., 693

Jenner, C. E., 1102

Jiang, G., 1010

Jiménez-Zurdo, J. I., 62, 96

Johnson, L., 742

Johnson, R. D., 742

Johnston, A. W. B., 228

Jonard, G., 520

Jones, J. B., 911, 1365

Jones, J. D. G., 465, 895

Jordá, M. A., 475

Jørgensen, B., 606

Jung, H. W., 136

Juwana, H., 895

Jwa, N.-S., 892

Kahmann, R., 1034

Kaku, H., 860

Kalavacharia, V., 1237

Kaló, P., 715

Kaneko, I., 1330

Karlsson, M.-B., 447

Katagiri, F., 1312

Katinakis, P., 14, 987

Kavroulakis, N., 14, 987

Keel, C., 232

Keen, N. T., 887

Kekarainen, T., 402

Kema, G. H. J., 1375

Khush, G., 869

Kijne, J. W., 268

Kikuchi, T., 374

Kim, C. Y., 470

Kim, D., 763

Kim, J. F., 1251

Kinane, J., 494

Kinscherf, T. G., 1263

Kiss, G. B., 715
Klessig, D. F., 118, 191, 347, 1015, 1380

Kloek, A. P., 1312

Knox, J. P., 413

Knox, M. R., 715

Kobori, T., 1195

Kodama, M., 742

Kohmoto, K., 742

Kõiv, V., 384, 447

Kondorosi, A., 62, 72, 96, 617, 799

Kondorosi, E., 62, 72, 799

Kouchi, H., 1156

Krake, L., 529

Kreuze, J. F., 366

Krishnan, H. B., 1010

Krüger, J., 1034

Kubo, Y., 374

Kumar, D., 347

Kunkel, B. N., 1312

Labavitch, J. M., 942

Laberge, S., 1271

Labidi, M., 1271

Lagudah, E. S., 334

Laigret, F., 1145

Lam, E., 191

Lam, S., 724

Lamers, G. E. M., 1170

Lange, J., 763

Langin, T., 287

Lapeyrie, F., 151

Lapidot, M., 673

Laplaze, L., 107, 113

Lau, G. W., 892

Laurent, F., 439

Laver, D., 325

Leach, J. E., 1322

Lebrun, M.-H., 217

Lebrun-Garcia, A., 821

Lecourieux-Ouaked, F., 821

Lee, S.-H., 470

Lee, S. Y., 470

Leferink-ten Klooster, H. B., 1385

Le Gall, O., 316

Lemcke, K., 787

Lettuce mosaic virus, green fluorescent protein and $\beta$-glucuronidase tagging, 316

Leung, H., 869, 1322

Leveau, J. H. J., 1243

Lim, C. O., 470

Lindow, S. E., 1243

Liu, Y., 118

Livingstone, K. D., 673

Llama-Palacios, A., 421

Lloret, J., 129

Loh, J. T., 1053

Lolium spp., endophyte mutualism, alkaloid protection against aphids, 1027

López, O., 1283

López-García, B., 837

López-Solanilla, E., 421

Lorito, M., 43

Lotus japonicus

- nitrogen-fixing symbiosis, calcium-binding protein homolog, 606

- nodulation, system based on transferase NodZ and acetyl transferase NolL, 475

—nodulin gene, symbiosis and embryonic tissue, 987

—-symbiosis in root epidermal cells, LjSym4 gene requirement, 1109

-uricase mRNA, analysis, 1156

Loubradou, G., 1034

Lough, T. J., 962

Lucas, W. J., 962, 1184 
Lugtenberg, B. J. J., 1170, 1177, 1340

Lycopersicon spp. (see also Tomato)

-L. esculentum, bacterial hypersensitivity, race 3 effect, 911

-L. pennellii, Xanthomonas oryzae race T3, gene-for-gene interaction, 1365

Lyon, G. D., 1092

Maclean, D. J., 929

Mäe, A., 384

Magnaporthe grisea

- conidiophore pattern mutation, LINE retrotransposon MGL, 892

-on rice: avirulence gene mapping, identification, 217; elicitor-responsive genes, analysis, 470; $m a g B$ gene, mutagenesis, 1214; resistance and lesion mimic mutants, 869

Maize

-anthracnose, pathogenicity mutants and enzyme-mediated integration, 1355

-cell apoptosis, Agrobacterium induction, 649

-Cochliobolus carbonum on, meiotic instability and virulence, 80

-smut: fungus growth and teliospore formation, in tumors, 1034; genes for filamentous growth, 781

Mäki-Valkama, T., 366

Mallory, A. C., 1027

Mangin, B., 6

Manners, J. M., 929

Manulis, S., 683

Marcos, J. F., 837

Marits, R., 447

Marqués, C., 23

Marston, M. F., 1266

Martín, M., 129

Martínez-Romero, E., 572, 796

Martin-Hernandez, A. M., 1301

Martini, I., 1109

Mathesius, U., 170, 617

Matsufuru, H., 860

Matsui, H., 210

Mavrodi, D. V., 1293

Mayda, E., 23

Mayorga, M. E., 781

McAlvin, C. B., 1053

McClean, P. E., 1237

McGeachy, K. D., 125

Medicago spp. (see also Alfalfa): syntenic genome regions, gene map, 715

-M. sativa, Sinorhizobium meliloti on, nod factors and stability in rhizosphere, 72

-M. truncatula: chitinase genes, mycorrhizae, nodulation, and infection, 763 ; nodule analysis, sequence tags, 62

Meletzus, D., 703

Melilotus alba, Sinorhizobium meliloti, proteome analysis in symbiosis, 995

Melton, R., 1301

Mendgen, K., 629

Mesorhizobium loti, on Lotus japonicus

—LjSym4 gene requirement, 1109

—nodulin gene and embryonic tissue, 987

-root-specific expression, calcium-binding protein, 606

Michelmore, R. W., 568

Miguel, E., 421

Milat, M.-L., 203

Miller, D. D., 1385

Miller, W. G., 1243

Minsavage, G. V., 911, 1365

Mise, K., 374, 1195
Mitsuhara, I., 210, 860

Mizen, S., 606

Montesano, M., 1092

Morant, M., 520

Morrissey, J. P., 1041

Moyer, J., 673

Muehlbauer, G. J., 159

Mulders, I. H. M., 1177, 1340

Mullineaaux, P., 529

Murai, N., 860

Murphy, J., 724

Mycosphaerella graminicola, on wheat, locus controls avirulence, 1375

Myers, J. R., 1237

Nachin, L., 882

Nakajima, Y., 860

Natera, S. H. A., 995

Natori, S., 860

Navarre, D. A., 1380

Nettleship, S. B., 1102

Netzler, N. E., 962

Nicholson, M. N., 606

Nicotiana spp. (see also tobacco): hypersensitivity, Cauliflower mosaic virus gene VI, agroinfiltration, 1275

- N. benthamiana: Agrobacterium-mediated transient expression, cover photo, April; Brome mosaic virus, virus recovery induced complementation and specific silencing, 247

$-N$. tabacum, hypersensitivity from

Pseudomonas syringae, 1346

Nikolskaya, A., 80

Nishijyo, T., 232

Nishimura, M., 892

Nodulation

-Casuarina glauca, prenodule cell display, 107

-lipochito-oligosaccharide factors, on vetch, 1385

-Lotus japonicus, fucosyl and acetyl transferases, 475

- Medicago truncatula, analysis and sequence tag expression, 62

-nodulin production, in soybean, apyrase expression, 1053

- pea, diamine oxidase and putrescine, 922

- Sinorhizobium meliloti, efficiency of nodulation, gene comparison, 583

- soybean, carbon metabolism and carbonic anhydrase, 14

Noguchi, M., 1330

Nomura, Y., 1330

Norman-Setterblad, C., 430

Notteghem, J. L., 217

Nuckles, E. M., 1355

Oat, Stagonospora avenae, enzymes and saponins in leaves, 1041

O'Gara, F., 232

Oger, P., 1081

Ohashi, Y., 210, 860

Ohshima, M., 860

Ohtsubo, N., 210

Oliver, R., 494

Olivier, J., 6

Olsthoorn, M. M. A., 808

Ophiostoma quercus, on elm, hydrophobin cerato-ulmin, 43

Orbach, M., 217

Osaki, T., 1195

Osborn, R. W., 54

Osbourn, A. E., 1041, 1301

Otani, H., 742
Overmars, H., 830, 1121

Ovtsyna, A. O., 799

Paiva, N. L., 551

Palanichelvam, K., 1081, 1275

Palva, E. T., 384, 430, 447, 1092

Panopoulos, N. J., 1346

Panter, S., 325

Paran, I., 673

Park, H. C., 470

Parker, J. E., 895

Parniske, M., 1109

Parrella, G., 43

Patil, S. S., 1071

Patriarca, E. J., 733

Pawlowski, K., 107, 113

Paynot, M., 238

Pea (see also Pisum spp.)

-nodule initiation, diamine oxidase and peroxidase involvement, 413

- transgenic lines, nodulation response and putrescine, 922

Pear

-Japanese, Alternaria alternata on, AK-toxin biosynthesis and pathogenicity, 975

- polygalacturonase inhibitor protein, tomato plant affected by, 942

Pehu, E., 366

Penicillium spp., on fruit, postharvest decay, hexapeptide activity, 837

Pérez-Payá, E., 837

Peronospora parasitica, on Arabidopsis, infection genes, 895

Phaseolus vulgaris

-anthracnose, resistance inheritance, trait loci, 287

-bacterial brown spot, growth and lesionforming ability of bacteria, transposon insertion, 1263

-Bean dwarf mosaic virus, hypersensitivity, BV1 protein determinant, 1184

- nitrogen fixation, symbiosis and the dnaJ locus, 1271

-nodule invasion and symbiosome differentiation during symbiosis, 733

—rust, resistance gene requirement, 1237

Phoelich, C. C., 1177

Phoma medicaginis, on alfalfa, resistance and resveratrol-glucoside, 551

Phytophthora infestans, on potato

- cyst germination protein, human mucin homology, 32

- gene encoding transcription factor, endochitinase coregulation, 1092

—inhibition by synthetic peptides, 847

Pirhonen, M., 384

Pisolithus tinctorius, on eucalypt, hypaphorine and auxins, interactions, 151

Pisum spp. (see also Pea): syntenic genome regions, gene map, 715

-P. sativum: Aphanomyces euteiches on, bioprotection using Glomus mosseae, 238; exudates, bacteria affected by, 637; Nod factors, cleaving activity in roots, chitinase effect, 799; root nodules, cathepsin B-like sequences, 778

Pitkin, J. W., 80

Poinsot, V., 72

Ponciano, G., 1322

Pontier, D., 19

Ponz, F., 1102

Popeijus, H., 830

Postharvest pathology, fruit, hexapeptide activity against fungi, 837 
Potato

- cyst nematode, pathogenicity factors, identification strategy, 830

- Erwinia carotovora and Phytophthora infestans, gene encoding a transcription factor, endochitinase, 1092

-late blight, cyst protein and human mucin homology, 32

- pathogen inhibition by synthetic peptides, 847

-potexviruses, cell-to-cell movement, ribonucleoprotein complex, 962

Potato mop-top virus, RNA movement and coat protein, resistant evidence, 125

Potato spindle tuber viroid, tomato protein kinase gene, characterization, 903

Potato virus A, recessive and dominant genes, diploid potato, 402

Potato virus $X$, coat protein and $25 \mathrm{~K}, 12 \mathrm{~K}$, and $8 \mathrm{~K}$ proteins, regulation, 599

Potato virus $Y$, transgenic resistance, homologous P1 sequences, 366

Powell, A. L. T., 942

Poza-Carrión, C., 421

Prinsen, E., 787

Pritsch, C., 159

Promé, J.-C., 72

Promoter-probe vectors, broad-host-range, $g f p$ and inaZ as reporter genes, 1243

Prusky, D., 887

Pseudomonas spp.: recombinant plasmids of, shuttle vectors, 232; on tomato, biocontrol for foot and root rot, 1177

-P. chlororaphis, tomato foot and root rot controlled by, root colonization, 1340

-P. fluorescens: damping-off controlled by, phenazine-1-carboxylic acid biosynthetic pathway, 1293; in rhizosphere, imaging and autofluorescent proteins, microbial community study, 1170

-P. syringae: on Arabidopsis, defense response and salicylic acid, jasmonic acid signal, 503; on Arabidopsis, hosts and nonhosts, temperature and phytotoxin synthesis, 951; on Arabidopsis, hypersensitivity in disease resistance, 277; on Arabidopsis, resistance and salicylic acid dependence, 877; on bean, phaseolotoxin-resistant ornithine carbamoyltransferase, thermal regulation, molecular analysis, 1071; on Nicotiana genotypes, hypersensitivity, 1346; on snap bean, growth and lesion-forming ability, transposon insertion, 1263; on soybean, cells challenged by inositol 1,4,5-trisphosphate, 563; on tomato, avirulence and virulence genes, mutation effect, 592; on tomato, growth and necrosis, avirulence gene effect, 568; on tomato, necrosis enhanced by functional genes, cover photo, May; on tomato, virulence promoted by avrRpt 2 gene, 1312

Puccinia spp., on wheat, resistance gene analogs, chromosomal segment, 334

Pugin, A., 821

Pyrenophora tritici-repentis, Ptr ToxA produced by, 456

Pythium ultimum, on pea, damping-off controlled by Pseudomonas fluorescens, biosynthetic pathway, 1293

Qin, L., 830

Quaedvlieg, N. E. M., 987

Radwanski, E. R., 673
Rajeshwari, R., 394

Ralstonia solanacearum

-on tomato, resistance, strain-specific locus, 6

-on tomato root, $h r p B$ and $h r p G$ regulatory genes, 259

Ranjeva, R., 693

Rathbun, E. A., 413

Rathjen, J. P., 568

Ray, S. K., 394

Red clover, cortical cell division induction, pea lectin gene, 268

Reddy, A. S. N., 847

Regenfelder, E., 1034

Renwick, A., 1293

Resistance

-Phaseolus vulgaris to Colletotrichum lindemuthianum, 287

- potato to Potato virus $Y$, transgenic P1 sequences, 366

Reviewers, acknowledgment, 3

Rezaian, A., 529

Rhizobium spp.: on alfalfa, intracellular nodules, 1204; plasmid marking, symbiosis stages, 1163 ; on red clover roots, pea lectin gene, 268; on white clover, fluorescent protein, cover photo, February; on white clover, nodulation induction, cortical cell activation, 170

- R. etli: fixN-reiterated genes, regulation, 1283; multiresistance genes, 572, 796; on Phaseolus vulgaris, nodule invasion and symbiosome differentiation, 733; vir gene homologs, self-transmissible plasmid, 1019

- R. leguminosarum: on bean, symbiosis and the dnaJ locus, 1271; lipo-chitin oligosaccharides, temperature regulation, 808; on Lotus japonicus, nodulation system, 475; on pea, diamine oxidase and peroxidase involvement, 413; on pea, Nod factors, hydrolyzed by chitinases, 799; on pea, nodulation and putrescine, $922 ;$ purMN genes, siderophore production, link, 228; on red clover, root nodule arrangement, cover photo, March; on vetch, mutant lacks nod genes, cover photo, July; vetch, root entry, Nod factor specificity, 754; on white clover, cortical cell division induction, cytokinin effect, 617

- R. tropici, guanine production and nodulation, temperature relation, 1228

Rhodes, D. J., 1293

Ribeiro, A., 113

Riccillo, P. M., 1228

Ricco, A., 733

Rice

-bacterial leaf blight, mutants and xylanase secretion, 394

-blast and bacterial blight, lesion mimic mutants and resistance, 869

-blast, elicitor-responsive genes, analysis, 470

- Pseudomonas syringae, defense-related genes, 342

Richards, K., 520

Ringer, J. P., 228

Ritzenthaler, C., 520

Rivilla, R., 129

Robinson, J. B., 637

Rodermel, S. R., 309

Rodríguez-Palenzuela, P., 421

Rolfe, B. G., 170, 617

Romero, D., 1283

Roncero, M. I. G., 359

Rossignol, M., 693
Roth, R., 439

Rouhara, I., 32

Rouppe van der Voort, J., 830

Roussis, A., 987

Rowley, K. B., 1071

Ruocco, M., 43

Rusu, A. G., 929

Saccharomyces cerevisiae, on dahlia, plant defensin binding site, 54

Salicylic acid

-induction required by NPR1 gene, transcription factors in defense genes, 191 -resistance required by, regulation, 877

Salzer, P., 763

Sánchez, F., 1102

Sánchez-Contreras, 129

Sanders, R., 297

Sarcophaga peregrina, on tobacco, peptide from and host resistance, 860

Sauviac, L., 6

Scala, A., 43

Scala, F., 43

Schardl, C. L., 1027

Schauser, L., 1109

Schlaman, H. R. M., 1163

Schmelzer, E., 32

Schmülling, T., 787

Schnider, U., 232

Schoelz, J. E., 1275

Schots, A., 830, 1121

Schultze, M., 72, 799

Seah, S., 334

Sentenac, H., 203

Seo, S., 210

Septoria lycopersici, on tomato, tomatinase gene, replacement, 1301

Sévignac, M., 287

Shababi, M., 1275

Shah, J., 191

Shan, L., 592

Shigaki, T., 563

Siegel, M. R., 1027

Sinorhizobium spp

-S. fredii, on soybean, cultivar-specific symbiont, 1010

-S. meliloti: on alfalfa, organogenetic pathways, molecular markers, 96; galactoglucan production, MucR requirement, 129; nod factors, $\mathrm{N}$ deacetylation, stability in alfalfa, rhizosphere, 72; nodulation formation efficiency, nitrogen fixation gene comparison, 583; pyruvate dehydrogenase genes, symbiotic induction, 483; root nodule symbiosis, gene disruption, 666; on white sweetclover, proteome analysis, symbiosis investigation, 995

Sivasithamparam, K., 334

Skøt, L., 606

Skroch, P., 287

Slezack, S., 238

Smant, G., 830

Soberón, M., 1283

Sohn, J., 629

Somers, D. A., 159

Sonti, R. V., 394

Soupène, E., 1204

Soybean

—apyrases, nodulin, 1053

-cells challenged by Pseudomonas syringae, inositol 1,4,5-trisphosphate, 563

-cultivar-specific symbiont, protein secretion system, 1010 
—nodules, carbon metabolism and carbonic anhydrase, 14

-root nodules, novel proteins and proteomics, 325

Spaink, H. P., 268, 475, 799, 808, 987, 1163

Spielmeyer, W., 334

Stacey, G., 1053

Staehelin, C., 72, 799

Stagonospora spp.

-S. avenae, on oat, enzymes and saponins, 1041

$-S$. nodorum, on wheat, trypsin produced in infection, characterization, 538

Stall, R. E., 911, 1365

Staskawicz, B. J., 568

Stephenson, S.-A., 929

Stevens, J. B., 228

Stewart, H. E., 1092

Stokvis, E., 808

Stougaard, J., 1109

Stussi-Garaud, C., 520

Stuurman, N., 475, 1163, 1170

Stylosanthes guianensis, anthracnose, pathogenicity and hypersensitivity, 929

Sudarshana, M. R., 1184

Sutherland, P., 962

Svistoonoff, S., 107

Symbiosis, Rhizobium etli, fixN-reiterated gene regulation, 1283

Syringolin A, on rice, phosphatase inhibitor, 342

Tajima, S., 1156

Takane, K., 1156

Takano, Y., 374

Tampakaki, A. P., 1346

Tanaka, A., 975

Tang, S., 465

Tang, X., 592

Tanksley, S. D., 1130

Taté, R., 733

Taylor, J. C., 1266

ten Have, A., 942

Teplitski, M., 637

Tharreau, D., 217

Thevissen, K., 54

Thibaud, J.-B., 203

Thomas, C. M., 465

Thomashow, L. S., 1293

Thomas-Oates, J. E., 475, 808

Thomson, R., 325

Thon, M. R., 1355

Thoquet, P., 6, 287

Tikhonovich, I. A., 799

Timmers, A. C. J., 1204

Timms-Wilson, T. M., 1293

Tobacco (see also Nicotiana spp.)

-bacterial and fungal pathogens, sarcotoxin IA effect, 860

-catalase, nitric oxide inhibition, ascorbate peroxidase, 1380

- defense reactions, cryptogein signal transduction, phosphoproteins, 821

- hypersensitivity, tomato gene effect, 465

-MAP kinase, gene encoding protein, interaction, 118

-MAP kinases, induction and defense signals, 347

- mitochondrial functions in cells, harpininduced cell death, 183

- phenotype hypersensitivity, cover photo, September

Tobacco mosaic virus

—on Arabidopsis thaliana, cell-to-cell movement and susceptibility, 1139

-hypersensitive peroxidase gene induction, spermine, 210

-on transgenic tobacco leaves, cover photo, January

Tokuyasu, K., 72

Tomato (see also Lycopersicon spp.)

-bacterial speck, growth and necrosis and avirulence genes, 568

-bacterial wilt, pathogenicity determinant, 703

-foot and root rot, biocontrol with bacteria, sss colonization gene, 1177

-Fusarium wilt, exopolygalacturonase and pgx4, 359

- pear fruit polygalacturonase inhibitor protein, colonization limits, 942

- protein kinase gene, Potato spindle tube viroid infection induced by, 903

-Ralstonia solanacearum: regulatory genes and infection stages, 259; resistance, strainspecific locus, 6

-resistance gene $S w-5$, tospovirus, root-knot nematode relation, 1130

-Septoria lycopersici on, tomatinase gene replacement, 1301

-Tomato mosaic virus, pathogen-induced gene and auxin, 23

- Tomato mottle virus and Bean dwarf mosaic virus relation, geminivirus movement proteins, 297

Tomato leaf curl virus, in tobacco, regulation in host, 529

Tomato mottle virus, Bean dwarf mosaic virus relation, geminivirus movement proteins, 297

Tomato spotted wilt virus, in Capsicum, resistance locus and gene, genetic mapping, 673

Toro, N., 583

Toth, I. K., 1092

Toxins

-beticolin, Cercospora beticola, 203

-Ptr ToxA, Pyrenophora tritici-repentis, 456

Trevaskis, B., 325

Trifa, Y., 191

Troussard, L., 62

Truchet, G., 1204

Tsuge, T., 975

Tuikkala, A., 384

Tuori, R. P., 456

Turnip crinkle virus, on Arabidopsis, AVR factor, coat protein, 1015

Turnip mosaic virus

-on Brassica napus, cylindrical inclusion bodies, cover photo, October

- cylindrical inclusion gene, pathogenic determinant in Brassica, 1102

Udvardi, M., 325

Ullrich, M. S., 951

Uromyces spp.

-U. appendiculatus, on bean, resistance gene analog cluster, 1237

-U. fabae, on Vicia faba, vitamin B1 biosynthesis genes in haustoria, 629

Ursin, V. M., 297

Ustilago maydis

-on corn, fungus growth and teliospore formation in tumors, 1034

-filamentous growth, role of $u b c 4$ and $u b c 5$ genes, 781

Vaillancourt, L. J., 1355
Valinsky, L., 683

Valkonen, J. P. T., 366, 402

Vallejos, C. E., 1365

Vance, C. P., 159

van der Biezen, E. A., 895

Van der Hoorn, R. A. L., 439

van Kan, J., 942

van Koert, P., 830

Van Montagu, M., 658

van Onckelen, H., 787

Vasse, J., 259, 1204

Vaubert, D., 62

Vera, P., 23

Verchot, J., 599

Verstappen, E. C. P., 1375

Vézina, L. P., 1271

Vicia spp.

-V. faba, rust, haustoria and vitamin B1

biosynthesis genes, 629

$-V$. hirsuta, root hairs, Nod factor specificity, 754

-V. sativa, root hairs, lipochitooligosaccharide nodulation factors, 1385

Vidal, S., 430

Vincent, J. L., 715, 778

Viruses, tospovirus, tomato, resistance gene $\mathrm{Mi}$, nematode-resistant homolog, 1130

Visser, J., 942

Voegele, R. T., 629

Vögeli-Lange, R., 763

Waalwijk, C., 1375

Wade, J., 232

Walker, S. A., 754

Walsh, J. A., 1102

Walsh, U., 232

Walton, J. D., 80

Wang, G.-L., 869

Wang, J.-F., 6

Wanner, G., 1034

Wäspi, U., 342

Watanabe, K. N., 402

Webb, J., 1109

Webb, K. J., 606

Wei, Z., 1251

Weinman, J. J., 170

Weissenmayer, B., 666

Weller, D. M., 1293

Wheat

-Fusarium head blight, defense response genes, induction in spikes, 159

-nematode and rust pathogens, resistance gene analogs, 334

- Septoria tritici leaf blotch, locus for avirulence control, 1375

White clover

-nodule initiation, specific markers, cover photo, June

- Rhizobium on, nodulation in lateral roots, 170

-Rhizobium leguminosarum, cortical cell division, cytokinin effect, 617

Widom, J., 1019

Wiemken, A., 763

Wijfjes, A. H. M., 475, 1163, 1170, 1177

Wilkinson, H. H., 1027

Willis, D. K., 1263

Willits, M. G., 503

Wisniewski, J.-P., 413, 922

Wobbe, K. K., 1015

Wolpert, T. J., 456

Wood, T. C., 1053

Wubben, J. P., 1041 
Xanthomonas spp.

-X. campestris: on pepper, cDNA genes, pathogenesis relation, 136; on tomato and pepper, T3 strain resistance, avirulence gene, 911

-X. oryzae: mutants, xylanase secretion, 394 on rice, avirulence genes and pathogen aggressiveness, 1322; on rice, resistance and lesion mimic mutants, 869; on tomato, gene-

for-gene interaction, race $\mathrm{T} 3$ and wild tomato, 1365
Xie, Z., 183

Xu, J.-R., 724

Xu, R., 1071

Yakoby, N., 887

Yamaguchi, I., 1330

Yamakawa, H., 210

Yang, Y., 599

Yeoman, K. H., 228

Yigit, E., 1015

Yin, Z., 869
Yoshida, T., 1330

Young, N. D., 1053

Yu, I., 277

Zeng, L., 869

Zhang, S., 118

Zhao, Y., 903, 1015

Zheng, L., 724

Zhou, J.-M., 191, 592 